\title{
17,000 \%/W Second-Harmonic Conversion Efficiency in Single-Crystalline Aluminum Nitride Microresonators
}

\author{
Alexander W. Bruch, ${ }^{1}$ Xianwen Liu, ${ }^{1}$ Xiang Guo, ${ }^{1}$ Joshua B. Surya, ${ }^{1}$ Zheng \\ Gong, ${ }^{1}$ Liang Zhang, ${ }^{2}$ Junxi Wang, ${ }^{2}$ Jianchang Yan, ${ }^{2}$ and Hong X. Tang 1 , 6 \\ ${ }^{1}$ Department of Electrical Engineering, Yale University, New Haven, CT 06511, USA \\ ${ }^{2} R \& D$ Center for Semiconductor Lighting, Institute of Semiconductors, Chinese Academy of Sciences, Beijing 100083, China
}

(Dated: November 9, 2018)

\begin{abstract}
High quality factor optical microcavities have been employed in a variety of material systems to enhance nonlinear optical interactions. While single-crystalline aluminum nitride microresonators have recently emerged as a low loss platform for integrated nonlinear optics such as four wave mixing and Raman lasing, few studies have investigated this material for second-harmonic generation. In this Letter, we demonstrate an optimized fabrication of dually-resonant phase-matched ring resonators from epitaxial aluminum nitride thin films. An unprecendented second-harmonic generation efficiency of $17,000 \% / \mathrm{W}$ is obtained in the low power regime and pump depletion is observed at a relatively low input power of $3.5 \mathrm{~mW}$. This poses epitaxial aluminum nitride as the highest efficiency second-harmonic generator among current integrated platforms.
\end{abstract}

On-chip optical nonlinearity has gained a significant interest for their applications in classical and quantum photonic information processing. Compared to their bulk counterparts, the tight optical confinement and facile device engineering in nanophotonic waveguides allow for greater experimental control over common nonlinear processes such as second-harmonic generation (SHG) [1-10], third harmonic generation (THG) [11-13], and photon-pair generation [1418]. These various nonlinear processes can be further combined, leading to applications of broadening the span of frequency combs [19-22], f-2f referencing [23], and all-optical control of energy transfer between optical modes [24]. Optical ring resonators have become a common modality to enhance the nonlinear effects. To date, various integrated platforms such as lithium niobate [2, 7, 8, 14, 18, 20, 25], silicon nitride $(\mathrm{SiN})[12,13,21,26,27]$, gallium nitride $(\mathrm{GaN})$ [4, 9, 10], and aluminum nitride (AIN) [5, 19, 28] have been employed to enhance the SHG efficiency beyond those seen in bulk crystals. AlN is particularly attractive for SHG for its large material band gap of $6.2 \mathrm{eV}$ and mature fabrication processing. Polycrystalline AlN microring resonators [5] have previously demonstrated state-of-the-art on-chip SHG conversion efficiency of $2500 \% / \mathrm{W}\left(0.025 \mathrm{~mW}^{-1}\right)$, owing to careful engineering of the phase-matching between the infrared and visible modes as well as efficient waveguide coupling for both pump and SHG light. However, the efficiency of this device was ultimately limited by the intrinsic optical quality $(Q)$ factor at both infrared (IR) and visible wavelengths. Increasing the material quality will ultimately reduce the optical loss at both fundamental and SHG wavelengths, allowing new record SHG efficiency.

Single-crystalline AIN has emerged as the likely successor to polycrystalline AlN photonics. As noted in previous studies, AlN epitaxially grown on sapphire substrates exhibit exceptionally high film quality and can achieve optical $Q$ factors on the order of $2 \times 10^{6}$ in the telecom band [29], much higher than those typically observed in polycrystalline AlN
[5, 11]. Currently, AlN-on-sapphire devices have been employed for linear and nonlinear phenomena at telecom IR wavelengths [30-32] as well as in the ultraviolet and visible regimes [33, 34]. Recently demonstrated UV SHG [35, 36] in AlN-on-sapphire devices was only observable using a pulsed pump while visible SHG in this platform [34] realized a low conversion efficiency due to the phase-mismatched condition. Lithography and etching challenges in processing thick (>1 $\mu \mathrm{m})$ AlN-on-sapphire films have proven as a barrier to achieving a fully-etched AlN waveguide [29-32], which is necessary to confine the phase-matched higher-order visible modes within the ring resonators.

In this Letter, we demonstrate a fabrication process for realizing fully-etched AlN-on-sapphire devices with a $1.1 \mu \mathrm{m}$ thickness. This process is then employed to fabricate ring resonators with both high optical $Q$ factors as well as a tunable phase-matching bandwidth of 1480-1640 nm. By optimizing the visible extraction waveguide and the coupling conditions of both infrared and visible modes, an on-chip SHG conversion efficiency of $17000 \% / \mathrm{W}$ was achieved in the low-power regime and a $\chi^{(2)}$ value of $6.2 \pm 0.4 \mathrm{pm} / \mathrm{V}$ is extracted for epitaxial AlN. Pump depletion was observed in the high-power regime, with an absolute conversion efficiency as high as $11 \%$.

We model the SHG process in a microring cavity possessing both an infrared and visible mode at angular frequencies $\omega_{I R}$ and $\omega_{v i s}$, respectively, each with internal loss rate, $\kappa_{0, I R(v i s)}$ and external coupling rate $\kappa_{c, I R(v i s)}$. The coupling strength between these two resonance modes is represented by the parameter $g$, which can be determined from the mode overlap between infrared and visible modes, the microring geometry, and the $\chi^{(2)}$ coefficient of AlN [5]. When pumping at a certain angular frequency, $\omega_{p}$, SHG efficiency in the non-depletion regime can be expressed in terms of these cavity coupling rates as well as the detuning between the infrared (visible) resonances from the pump, $\delta_{I R}=\omega_{I R}-\omega_{p}$ $\left(\delta_{v i s}=\omega_{v i s}-2 \omega_{p}\right)$, as

$$
\eta_{S H G}=\frac{P_{S H G}}{P_{p}^{2}}=g^{2} \frac{\hbar \omega_{v i s}}{\left(\hbar \omega_{p}\right)^{2}} \frac{2 \kappa_{c, v i s}}{\delta_{v i s}^{2}+\kappa_{v i s}^{2}}\left(\frac{2 \kappa_{c, I R}}{\delta_{I R}^{2}+\kappa_{I R}^{2}}\right)^{2}
$$


where $P_{S H G}$ and $P_{p}$ represent the on-chip SHG and pump power, respectively, and $\kappa_{I R(v i s)}$ is the total coupling rate $\kappa_{I R(v i s)}=\kappa_{c, I R(v i s)}+\kappa_{0, I R(v i s)}$. Under ideal phase matching conditions, the microring geometry is optimized such that $\delta_{I R}=\delta_{v i s}=0$ and $\omega_{v i s}=2 \omega_{I R}=2 \omega_{p}$. Fulfilling this dualresonance condition, the SHG conversion efficiency can be reduced to

$$
\eta_{S H G}=\frac{P_{S H G}}{P_{p}^{2}}=\frac{16 g^{2}}{\hbar \omega_{p}} \frac{\kappa_{c, v i s}}{\left(\kappa_{c, v i s}+\kappa_{0, v i s}\right)^{2}} \frac{\kappa_{c, I R}^{2}}{\left(\kappa_{c, I R}+\kappa_{0, I R}\right)^{4}}
$$

According to Eq. 2, maximum $\eta_{S H G}$ can be achieved when both the IR and visible modes are critically coupled $\left(\kappa_{c}=\kappa_{0}\right)$ and the SHG efficiency is dependent only on the intrinsic device parameters. As noted in our previous report in polycrystalline AIN [5], it is practically difficult to simultaneously achieve critical coupling for both modes in the phase-matched geometry and the maximum conversion efficiency is often limited by the cavity coupling rate.

In this experiment, single-crystalline AlN films are grown on c-plane sapphire by metal-organic vapor deposition (MOCVD) to a nominal thickness of $1.1 \mu \mathrm{m}$. The AlNon-sapphire platform is quite insulating compared to typical silicon-based substrates and suffers from significant charging effects in our $100 \mathrm{kV}$ electron-beam lithography process (Raith EBPG 5000+). While metal masks are typically used for increasing the conductivity of insulating substrates, many are not compatible with typical negative-tone resists such as hydrogen silsesquioxane (HSQ) and/or require a twostep etching process that ultimately limits the process selectivity [29]. To mitigate charging effects, we first spin a conventional FOx-16 HSQ mask followed by $300 \mathrm{~nm}$ poly(4styrenesulfonic acid) (PSSA) before sputtering $10 \mathrm{~nm}$ gold. PSSA acts as a water-soluble sacrificial layer between the conductive gold layer and FOx-16 resist, allowing sufficient charge transfer to the HSQ layer and is easily removed during the development process [37]. Patterns are then developed in a $25 \%$ TMAH solution to maintain a high contrast. The patterns are then transferred to the AlN via an optimized $\mathrm{Cl}_{2} / \mathrm{BCl}_{3} / \mathrm{Ar}$ inductively coupled plasma (ICP) etch with a selectivity of 2.7:1 between AIN and FOx-16. Devices are then encapsulated in $1.0 \mu \mathrm{m}$ plasma-enhanced chemical vapor deposition (PECVD) $\mathrm{SiO}_{2}$ and cleaved prior to measurement.

This process provides a simplified route for AlN-onsapphire fabrication, requiring a single masking step compared to the two-step processes previously reported in Ref. [29]. The thicker FOx-16 mask and the high etching selectivity allow up to $1.4 \mu \mathrm{m}$ of AlN to be fully etched, compared to $1.0 \mu \mathrm{m}$ demonstrated previously. This fully etched structure is particularly important for tight confinement of the visible mode. Finite element method simulations indicate that the second-harmonic mode exhibits $1.2 \mathrm{~dB} / \mathrm{cm}$ radiation loss when leaving a $200 \mathrm{~nm}$ unetched AlN layer (radius $=30 \mathrm{um}$, height $=1 \mu \mathrm{m}$, width $=1.2 \mu \mathrm{m}$ ), whereas the radiation loss is negligible in the fully-etched structure.

Fig. 1(a) depicts the final fabricated devices, where a 900 nm-wide bus waveguide is coupled to a microring resonator (a)

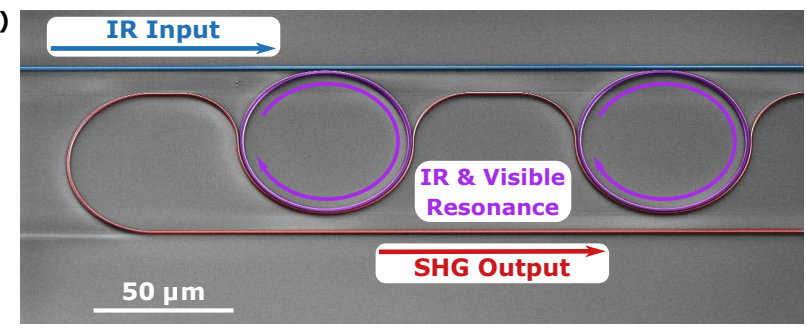

(b)
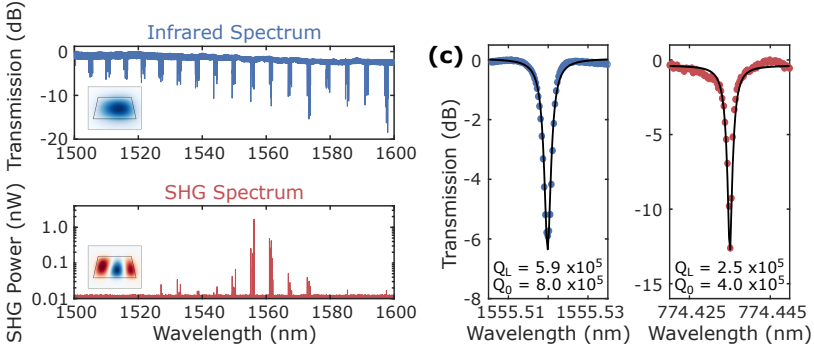

Figure 1. AlN device design and performance. (a) Scanning electron microscope (SEM) micrograph of a fabricated AlN-on-sapphire device before encapsulation in $\mathrm{PECVD} \mathrm{SiO}_{2}$. A straight bus waveguide couples telecom IR light (blue) into cascaded microring resonators exciting a dual resonance at infrared and visible wavelengths (purple). The wrap around waveguide efficiently extracts near-visible SHG light (red) from the microrings and passes the output to an onchip WDM [5, 38]. (b) Typical spectra for phase-matched microring resonators. Sweeping an IR laser (blue) shows multiple resonance dips corresponding to the cascaded microring structure. Phase matched resonances near $1555-1560 \mathrm{~nm}$ can be identified by peaks in SHG spectrum (red). The insets highlight the IR $\mathrm{TM}_{0,0}$ pump mode and the phase matched $\mathrm{TM}_{2,0}$ SHG mode, respectively. (c) Resonance spectra of infrared $\mathrm{TM}_{0,0}$ and near-visible $\mathrm{TM}_{2,0}$ modes with loaded and intrinsic $Q$ values shown in the insets.

with a waveguide width of 1.2-1.3 $\mu \mathrm{m}$. A thinner waveguide wrapped around the ring is employed to efficiently extract the near-visible light from the phase-matched ring resonator. The near-visible extraction waveguide width is tapered from 175 to $125 \mathrm{~nm}$ at a constant coupling gap. As a result, the fundamental mode of the extraction waveguide can be sufficiently matched to the near-visible $\mathrm{TM}_{2,0}$ ring mode while minimally perturbing the infrared $\mathrm{TM}_{0,0}$ ring mode. Seven ring resonators share the same IR bus and visible drop waveguides to account for fabrication imperfections across the AIN chip. The infrared and visible waveguides rejoin in an onchip wavelength division multiplexer (WDM), while an offchip WDM separates the infrared and near-visible light for simultaneous characterization [5, 19, 38].

Microring resonances are observed by sweeping the wavelength of an infrared external cavity tunable diode laser (Santec TSL 710), as shown in Fig. 1 (b). In phase-matched devices, cavity resonances in the infrared spectrum occur simultaneously with peaks observed in the corresponding visible spectrum, indicative of the dual-resonance process. Fig. 11(c) shows typical resonance spectra at $1550 \mathrm{~nm}$ and $775 \mathrm{~nm}$. The infrared resonance is slightly undercoupled with a loaded $Q$ factor $\left(Q_{L}\right)$ of $5.9 \times 10^{5}$, while the second-harmonic mode is nearly critically coupled with a $Q_{L}$ of $2.5 \times 10^{5}$. We note that the extinction ratio of the infrared resonance increases when 
scanning from shorter to longer wavelengths in Fig. 1 b) with minimal impact on the optical $Q$ factors. These optical $Q$ factors are more than doubled compared to polycrystalline AIN devices with a similar geometry [5] and are of similar order of magnitude compared to previous AlN-on-sapphire devices [29], which had larger dimensions and were partially etched, inducing lower sidewall scattering loss but higher visible radiation loss as described earlier.

We then optimize the SHG wavelength by varying the width of the AlN ring waveguide, as shown in Fig. 2(a). By scanning the ring width from 1.2 to $1.3 \mu \mathrm{m}$, SHG can be observed over the entire range of our tunable laser (1480-1640 nm). The experimental SHG wavelength agrees remarkably well with the calculated results from a FIMMWAVE simulation. We then optimize the extraction efficiency at a particular wavelength by varying the coupling gap of the visible extraction waveguide. Fig. 2(b) displays the SHG power collected as a function of both the phase-matching width and visible coupling gap in the C-band erbium-doped fiber amplifier (EDFA) window of 1540-1560 nm (gray band in Fig. 2(a)). The observed SHG power is very sensitive to the ring waveguide width, which is the main determinant of the phase-matching condition between the IR and visible modes. In contrast, a variety of visible coupling gaps are able to extract the SHG power, due to the adoption of the wrapped extraction waveguides. From an input pump power of $190 \mu \mathrm{W}$, a maximum external SHG power of $20 \mathrm{nW}$ can be collected from a ring waveguide width of $1.24 \mu \mathrm{m}$ and a visible coupling gap of $0.65 \mu \mathrm{m}$, whereas the SHG output decreases for visible coupling gaps below 0.60 $\mu \mathrm{m}$. Scanning reference devices at the SHG wavelength reveals that the $0.60-0.65 \mu \mathrm{m}$ gaps are nearly critically coupled, whereas devices with visible coupling gaps of 0.50-0.55 $\mu \mathrm{m}$ are overcoupled and exhibit diminished SHG output as predicted by Eq. 2.

At low pump levels, SHG output power is known to increase quadratically with the pump power and deviates from this behavior with high pump power due to pump depletion. Fig. 2(c) depicts the pump power dependence of the SHG output power in a device with a $1.24 \mu \mathrm{m}$ width and a 0.65 $\mu \mathrm{m}$ visible coupling gap. At low pump power, the variation of the SHG output power is close to the predicted quadratic dependence on the pump power, with a fitted slope of 1.94 \pm 0.02 , whereas the SHG output power begins to deviate from the quadratic behavior at high pump power. The lower inset to Fig. 2(c) shows the absolute conversion efficiency $\left(P_{S H G} / P_{P}\right)$, which deviates greater than $50 \%$ from the predicted behavior when the on-chip pump power is greater than $3.5 \mathrm{~mW}$ and saturates to $11 \%$ when $P_{P}$ is greater than $35 \mathrm{~mW}$. This deviation from the quadratic power dependence is observed in the high power regime, which indicates significant pump depletion and ultimately limits the maximum SHG efficiency [3, 5]. Looking to Eq. 1, we notice that $P_{S H G}$ varies linearly with $P_{p}^{2}$ with a slope equal to the SHG efficiency $\eta$; the upper inset to Fig. 2 (c) shows this relation and a SHG efficiency of $14,500 \pm 200$ $\% / \mathrm{W}$ for this device is found via a linear fit of the low power regime. We then use the experimentally measured values of $\kappa_{0}$ and $\kappa_{c}$ at infrared and near-visible regimes to derive a nonlinear coupling strength $g / 2 \pi=0.12 \mathrm{MHz}$. This nonlinear
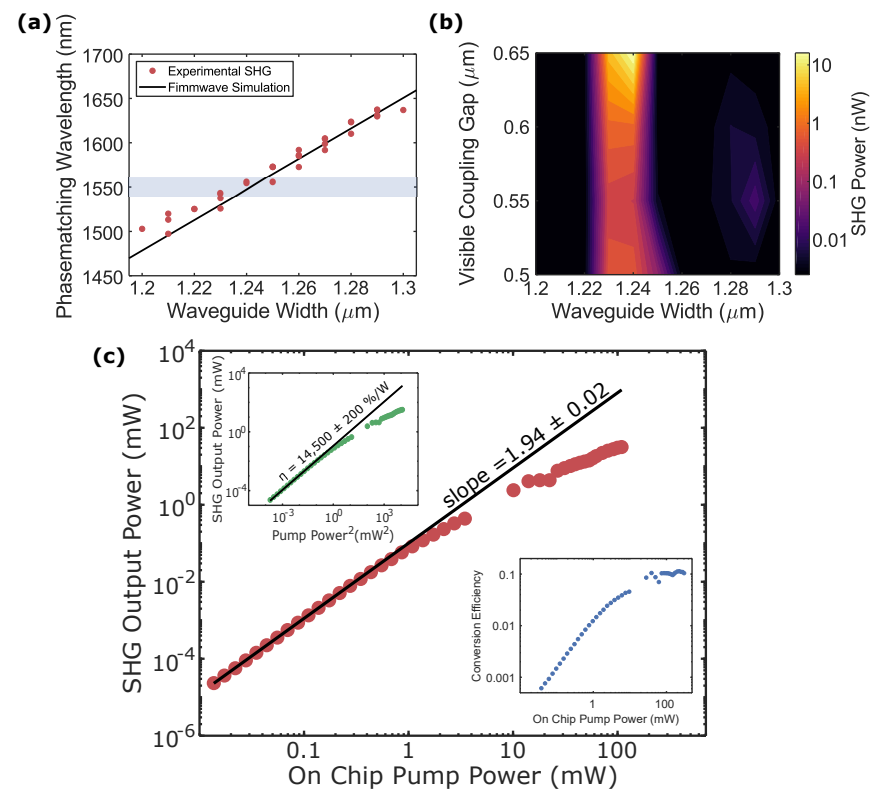

Figure 2. Geometrical optimization of SHG performance. (a) Experimentally recorded and simulated SHG wavelength for devices with ring widths of 1.2-1.3 $\mu \mathrm{m}$. The two data sets exhibit a high degree of agreement. (b) Heat map of experimental SHG power as a function of the waveguide width and the visible coupling gap for the EDFA window (gray band in (a)). Maximum SHG power of $20 \mathrm{nW}$ is observed for waveguide width $1.24 \mu \mathrm{m}$ and visible coupling gap of 0.65 $\mu \mathrm{m}$. (c) Pump power dependence of SHG output power. Experimental data (red) is plotted with a linear fit of the low power region (solid line, slope $=1.94 \pm 0.02$ ). The lower inset (blue) shows the absolute conversion efficiency over the same pump power. The upper inset (green) shows the variation of SHG output power versus $P_{p}^{2}$. A linear fit of the low power region shows a $\mathrm{SH}$ conversion efficiency of $14,500 \pm 200 \% / \mathrm{W}$.

coupling strength, mode overlap factors calculated by FEM simulations, and relevant device parameters [5] are used to derive a $\chi^{(2)}$ value of $6.2 \pm 0.4 \mathrm{pm} / \mathrm{V}$ for the single-crystalline AlN device platform. This value is quite close to the $8.6 \mathrm{pm} / \mathrm{V}$ recently measured in thin films of MOCVD AlN at $1064 \mathrm{~nm}$ [39] and is nearly three times higher than previously reported values for polycrystalline AIN [40, 41].

Finally, we look to Eq. 2 to optimize the SHG efficiency. As noted earlier, the SHG efficiency is optimized when the fundamental and second-harmonic resonances are both critically coupled. However, this proves difficult in practice as one (or both) resonances may deviate from critical coupling when varying the device geometries. Fig. 3 (a) shows the effect of cavity coupling on the SHG efficiency (Eq. 2) using the derived $g / 2 \pi=0.12 \mathrm{MHz}$ obtained above and experimentally measured intrinsic loss rates of $\kappa_{0, I R} / 2 \pi=240 \mathrm{MHz}$ and $\kappa_{0, v i s} / 2 \pi=970 \mathrm{MHz}$ for infrared and near-visible modes, respectively. The SHG efficiency is maximized when both modes are critically coupled $\left(\kappa_{0} / \kappa_{c}=1\right)$ and decreases as the modes become over- or under-coupled.

The device used in the previous pump-power experiment in Fig. 2] was nearly critically coupled for the near-visible resonance, but under-coupled for the IR resonance $\left(\kappa_{0} / \kappa_{c}>1\right)$, 
(a)

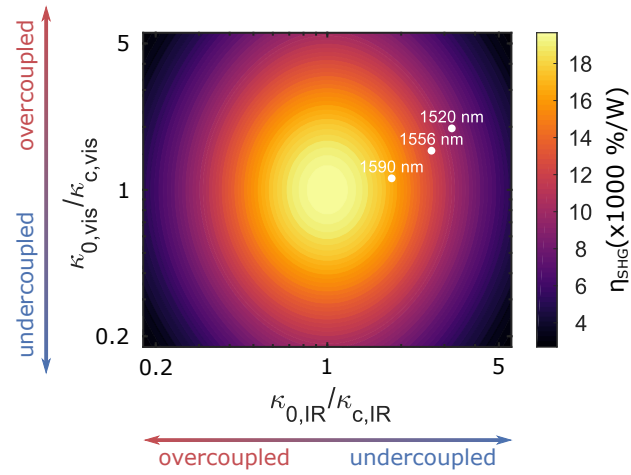

(b)

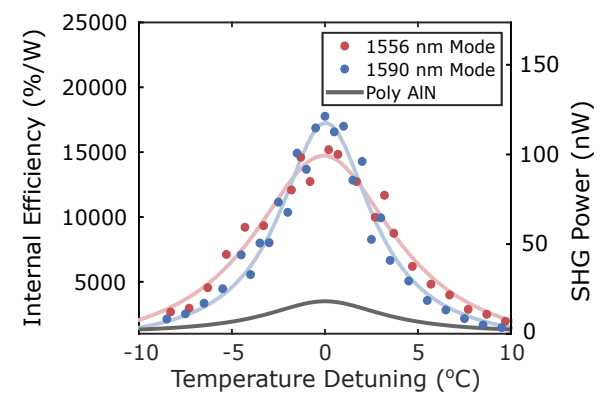

Figure 3. Optimal coupling and temperature for SHG. (a) Simulated SHG efficiency from Eq. 2 is parametrically solved with the cavity coupling rate $\kappa_{c}$ at IR and visible wavelengths. A peak conversion efficiency of $20,000 \% / \mathrm{W}$ can be achieved at critical coupling for both modes. Experimental $\kappa_{0} / \kappa_{I}$ are displayed for selected wavelengths. (b) Temperature dependent SHG efficiency of an optical mode at $1590 \mathrm{~nm}$ (blue) and $1556 \mathrm{~nm}$ (red). A Lorentzian fit (solid line) is applied to the experimental data (circles). A maximum internal SHG conversion efficiency of $17,000 \% / \mathrm{W}$ and $15,000 \% / \mathrm{W}$ are observed for $1590 \mathrm{~nm}$ and $1556 \mathrm{~nm}$ modes, respectively. Simulated data from polycrystalline AlN with experimental parameters from [5] is shown for reference (black line).

yet can still achieve a relatively high conversion efficiency of $14,500 \pm 200 \% / \mathrm{W}$. We notice that extinction ratio of the IR resonance increases when scanning from short to long wavelengths (Fig. 1(b)); the cavity coupling rate can be increased and a more ideal value for the SHG efficiency can be obtained by choosing a pump mode at a longer wavelength. Experimental values of $\kappa_{0} / \kappa_{c}$ are obtained at various wavelengths and are superimposed on the contour plot in Fig. 3(a), which indeed suggest that using a mode at $1590 \mathrm{~nm}$ may increase the experimental SHG efficiency. We systematically optimize the SHG output of optical modes at $1590 \mathrm{~nm}$ and $1556 \mathrm{~nm}$ by varying the global temperature of the chip to align the wavelengths of infrared and visible resonances, as shown in Fig. 3 b). With an input pump power (measured in the input fiber) of $260 \mu \mathrm{W}$, a maximum SHG output power of $120 \mathrm{nW}$ and $110 \mathrm{nW}$ are collected for $1590 \mathrm{~nm}$ and $1556 \mathrm{~nm}$ modes, respectively. After considering the transmission of $22 \%$ and 10 $\%$ for IR and visible wavelengths, respectively, an on-chip (internal) SHG efficiency of $17,000 \% / \mathrm{W}$ and $15,000 \% / \mathrm{W}$ can be calculated for each mode. The observed SHG efficiencies are in good agreement with the theoretical values calculated in Fig. [3 a) for both modes as well as that calculated in Fig. 2. (c) for the $1556 \mathrm{~nm}$ mode without fine thermal tuning. We calculate a $\pm 3000 \% / \mathrm{W}$ uncertainty in the calculated conversion efficiency, arising mainly from the $\pm 10 \%$ relative error in measuring the absolute transmission for both infrared and visible wavelengths as well as amplitude noise from the high-sensitivity visible photodetector. This internal conversion efficiency is a seven-fold improvement compared to previous polycrystalline AlN devices [5], manifesting our result as a new record for SHG among current integrated platforms. We attribute this record high SHG efficiency to the greatly increased optical $Q$ factors at both infrared and near-visible wavelengths compared to previous device platforms as well as a relatively high material $\chi^{(2)}$ value obtained in this work. We do not believe a defect-related SHG proces plays a role at this time, as the effective $\chi^{(2)}$ value for surface charge SHG is typically one to two orders of magnitude less than that observed in AlN [12, 42].

In conclusion, we demonstrate a fabrication process to realize high quality AlN-on-sapphire nanophotonic devices for integrated nonlinear photonic applications. In the low pump power regime, an on-chip SHG conversion efficiency of $17,000 \% / \mathrm{W}\left(0.17 \mathrm{~mW}^{-1}\right)$ is recorded, which is the new state-of-the-art thus observed among integrated platforms. At high pump power, pump depletion is observed at a relatively low pump power of $3.5 \mathrm{~mW}$. The demonstrated high SHG efficiency paves the way for applications including low loss coherent frequency conversion [38], on-chip comb selfreferencing, as well as more efficient correlated photon-pair sources [16].

This work is supported by DARPA SCOUT (W31P4Q15-1-0006) and ACES programs as part of the Draper-NIST collaboration (HR0011-16-C-0118). H.X.T. acknowledges support funding from an AFOSR MURI grants (FA9550-151-0029), LPS/ARO grant (W911NF-18-1-0020), NSF EFRI grant (EFMA-1640959) and David and Lucile Packard Foundation.
[1] M. Cazzanelli, F. Bianco, E. Borga, G. Pucker, M. Ghulinyan, E. Degoli, E. Luppi, V. Véniard, S. Ossicini, D. Modotto, S. Wabnitz, R. Pierobon, and L. Pavesi, Nat. Mater. 11, 148 (2011).

[2] L. Chang, Y. Li, N. Volet, L. Wang, J. Peters, and J. E. Bowers, Optica 3, 531 (2016)

[3] J. U. Fürst, D. V. Strekalov, D. Elser, M. Lassen,
U. L. Andersen, C. Marquardt, and G. Leuchs, Phys. Rev. Lett. 104, 153901 (2010)

[4] M. Gromovyi, J. Brault, A. Courville, S. Rennesson, F. Semond, G. Feuillet, P. Baldi, P. Boucaud, J.-Y. Duboz, and M. P. De Micheli, Opt. Express 25, 23035 (2017)

[5] X. Guo, C.-L. Zou, and H. X. Tang, Optica 3, 1126 (2016)

[6] Z. Lin, X. Liang, M. Lončar, S. G. Johnson, and A. W. Ro- 
driguez, Optica 3, 233 (2016)

[7] C. Wang, X. Xiong, N. Andrade, V. Venkataraman, X.-F. Ren, G.-C. Guo, and M. Lončar, Opt. Express 25, 6963 (2017)

[8] R. Wolf, I. Breunig, H. Zappe, and K. Buse, Opt. Express 25, 29927 (2017).

[9] C. Xiong, W. Pernice, K. K. Ryu, C. Schuck, K. Y. Fong, T. Palacios, and H. X. Tang, Opt. Express 19, 10462 (2011).

[10] I. Roland, M. Gromovyi, Y. Zeng, M. El Kurdi, S. Sauvage, C. Brimont, T. Guillet, B. Gayral, F. Semond, J. Y. Duboz, M. de Micheli, X. Checoury, and P. Boucaud, Sci. Rep. 6, 34191 (2016)

[11] J. B. Surya, X. Guo, C.-L. Zou, and H. X. Tang, Optica 5, 103 (2018)

[12] J. S. Levy, M. A. Foster, A. L. Gaeta, and M. Lipson, Opt. Express 19, 11415 (2011).

[13] L. Wang, L. Chang, N. Volet, M. H. P. Pfeiffer, M. Zervas, H. Guo, T. J. Kippenberg, and J. E. Bowers, Laser Photon. Rev. 10, 631 (2016)

[14] R. Luo, H. Jiang, S. Rogers, H. Liang, Y. He, and Q. Lin, Opt. Express 25, 24531 (2017)

[15] S. Clemmen, K. P. Huy, W. Bogaerts, R. G. Baets, P. Emplit, and S. Massar, Opt. Express 17, 16558 (2009).

[16] X. Guo, C.-1. Zou, C. Schuck, H. Jung, R. Cheng, and H. X. Tang, Light Sci. Appl. 6, e16249 (2016)

[17] J. E. Sharping, K. F. Lee, M. A. Foster, A. C. Turner, B. S. Schmidt, M. Lipson, A. L. Gaeta, and P. Kumar, Opt. Express 14, 12388 (2006).

[18] H. Jin, F. Liu, P. Xu, J. Xia, M. Zhong, Y. Yuan, J. Zhou, Y. Gong, W. Wang, and S. Zhu, Phys. Rev. Lett. 113, 103601 (2014).

[19] X. Guo, C.-L. Zou, H. Jung, Z. Gong, A. Bruch, L. Jiang, and H. X. Tang, (2017) arXiv:1704.04264.

[20] J. Lin, Y. Xu, J. Ni, M. Wang, Z. Fang, L. Qiao, W. Fang, and Y. Cheng, Phys. Rev. Appl. 6, 014002 (2016).

[21] X. Xue, F. Leo, Y. Xuan, J. A. Jaramillo-Villegas, P.-H. Wang, D. E. Leaird, M. Erkintalo, M. Qi, and A. M. Weiner, Light Sci. Appl. 6, e16253 (2017)

[22] A. S. Kowligy, A. Lind, D. D. Hickstein, D. R. Carlson, H. Timmers, N. Nader, F. C. Cruz, G. Ycas, S. B. Papp, and S. A. Diddams, Opt. Lett. 43, 1678 (2018)

[23] D. D. Hickstein, H. Jung, D. R. Carlson, A. Lind, I. Coddington, K. Srinivasan, G. G. Ycas, D. C. Cole, A. Kowligy, C. Fredrick, S. Droste, E. S. Lamb, N. R. Newbury, H. X. Tang, S. A. Diddams, and S. B. Papp, Phys. Rev. Appl. 8, 014025 (2017)

[24] X. Guo, C.-L. Zou, L. Jiang, and H. X. Tang, (2018), arXiv:1804.06921.

[25] C. Wang, M. J. Burek, Z. Lin, H. A. Atikian, V. Venkataraman, I.-C. Huang, P. Stark, and M. Lončar,
Opt. Express 22, 30924 (2014)

[26] T. Ning, H. Pietarinen, O. Hyvärinen, R. Kumar, T. Kaplas, M. Kauranen, and G. Genty, Opt. Lett. 37, 4269 (2012).

[27] T. Ning, H. Pietarinen, O. Hyvärinen, J. Simonen, G. Genty, and M. Kauranen, Appl. Phys. Lett. 100, 161902 (2012)

[28] W. H. P. Pernice, C. Xiong, C. Schuck, and H. X. Tang, Appl. Phys. Lett. 100, 223501 (2012)

[29] X. Liu, C. Sun, B. Xiong, L. Wang, J. Wang, Y. Han, Z. Hao, H. Li, Y. Luo, J. Yan, T. Wei, Y. Zhang, and J. Wang, Opt. Express 25, 587 (2017).

[30] X. Liu, C. Sun, B. Xiong, L. Wang, J. Wang, Y. Han, Z. Hao, H. Li, Y. Luo, J. Yan, T. Wei, Y. Zhang, and J. Wang, Optica 4, 893 (2017)

[31] X. Liu, C. Sun, B. Xiong, L. Wang, J. Wang, Y. Han, Z. Hao, H. Li, Y. Luo, J. Yan, T. Wei, Y. Zhang, and J. Wang, ACS Photonics 5, 1943 (2018)

[32] X. Liu, C. Sun, B. Xiong, J. Wang, L. Wang, Y. Han, Z. Hao, H. Li, Y. Luo, J. Yan, T. bo Wei, Y. Zhang, and J. Wang, Opt. Lett. 41, 3599 (2016).

[33] T.-J. Lu, M. Fanto, H. Choi, P. Thomas, J. Steidle, S. Mouradian, W. Kong, D. Zhu, H. Moon, K. Berggren, J. Kim, M. Soltani, S. Preble, and D. Englund, Opt. Express 26, 11147 (2018)

[34] X. Liu, C. Sun, B. Xiong, L. Wang, J. Wang, Y. Han, Z. Hao, H. Li, Y. Luo, J. YAN, T. Wei, Y. Zhang, and J. Wang, in Conf. Lasers Electro-Optics (OSA, Washington, D.C., 2017) p. FTu3D.1.

[35] M. L. Fanto, J. A. Steidle, t.-j. J. Lu, S. F. Preble, D. R. Englund, c. C. Tison, a. matthew Smith, g. A. Howland, kathy-anne Soderberg, P. Alsing, G. A. Howland, K. Soderberg, and P. M. Alsing, in Front. Opt. 2016 (OSA, Washington, D.C., 2016) p. FTh5G.6.

[36] T. Troha, M. Rigler, D. Alden, I. Bryan, W. Guo, R. Kirste, S. Mita, M. D. Gerhold, R. Collazo, Z. Sitar, and M. Zgonik, Opt. Mater. Express 6, 2014 (2016)

[37] M. Rooks, "Conducting layer for HSQ resist,"

[38] X. Guo, C.-L. Zou, H. Jung, and H. X. Tang, Phys. Rev. Lett. 117, 123902 (2016)

[39] A. Majkić, A. Franke, R. Kirste, R. Schlesser, R. Collazo, Z. Sitar, and M. Zgonik, Phys. Status Solidi Basic Res. 254 (2017), 10.1002/pssb.201700077

[40] P. Gräupner, J. C. Pommier, A. Cachard, and J. L. Coutaz, J. Appl. Phys. 71, 4136 (1992)

[41] C. Xiong, W. H. P. Pernice, and H. X. Tang, Nano Lett. 12, 3562 (2012).

[42] M. W. Puckett, R. Sharma, H.-H. Lin, M.-h. Yang, F. Vallini, and Y. Fainman, Opt. Express 24, 16923 (2016). 